\title{
Effects of Fine Particulate Matter on Daily Mortality for Specific Heart Diseases in Japan
}

\author{
Kayo Ueda, MD; Hiroshi Nitta, DHSc; Masaji Ono, DHSc
}

(Circ J 2009; 73: 1248-1254)

\begin{abstract}
The author apologizes for errors which appeared in the Table 1, page 1249, Vol. 73. The daily max/min numbers for cardiac deaths were those for aged 75 and over, and the daily mean numbers were those by age categories (which were one third of correct numbers). The percentages of age categories in Fukuoka, Kawasaki, Sakai, and Sendai were incorrect. The correct version of Table 1 is provided below.
\end{abstract}

Table 1. Demographic Statistics of Mortality From Heart Disease in 9 Areas of Japan, 2002-2004

\begin{tabular}{|c|c|c|c|c|c|c|c|c|c|}
\hline & Tokyo & Osaka & Nagoya & Kobe & Sapporo & Fukuoka & Kawasaki & Sakai & Sendai \\
\hline Total no. of cardiac deaths & 28,765 & 9,395 & 8,256 & 5,178 & 4,767 & 3,146 & 3,107 & 2,679 & 2,604 \\
\hline Rates per $100,000^{*}$ & 115.4 & 119.8 & 125.5 & 114.4 & 85.8 & 76.5 & 80.4 & 107.6 & 85.4 \\
\hline Daily mean no. of cardiac deaths ( $\min -\max )$ & $\begin{array}{r}26.2 \\
(6-53)\end{array}$ & $\begin{array}{r}8.6 \\
(1-22)\end{array}$ & $\begin{array}{r}7.5 \\
(0-25)\end{array}$ & $\begin{array}{r}4.7 \\
(0-15)\end{array}$ & $\begin{array}{r}4.3 \\
(0-13)\end{array}$ & $\begin{array}{r}2.9 \\
(0-12)\end{array}$ & $\begin{array}{r}2.8 \\
(0-9)\end{array}$ & $\begin{array}{r}2.4 \\
(0-10)\end{array}$ & $\begin{array}{r}2.4 \\
(0-10)\end{array}$ \\
\hline \multicolumn{10}{|l|}{ Age $(\%)$} \\
\hline $0-64$ & 14.7 & 16.2 & 17.4 & 19.3 & 13.2 & 19.8 & 14.4 & 16.5 & 16.0 \\
\hline $65-74$ & 18.3 & 20.8 & 19.6 & 17.2 & 18.4 & 20.2 & 17.6 & 21.3 & 17.2 \\
\hline $75+$ & 67.1 & 63.0 & 63.0 & 63.5 & 68.4 & 59.9 & 68.0 & 62.2 & 66.9 \\
\hline \multicolumn{10}{|l|}{$\operatorname{Sex}(\%)$} \\
\hline Men & 50.9 & 50.9 & 51.9 & 50.0 & 47.7 & 53.8 & 47.4 & 50.1 & 50.8 \\
\hline Women & 49.1 & 49.1 & 48.1 & 50.0 & 52.3 & 46.2 & 52.6 & 49.9 & 49.2 \\
\hline \multicolumn{10}{|l|}{ Specific cardiac deaths (\%) } \\
\hline AMI & 20.8 & 22.4 & 21.7 & 27.7 & 28.7 & 36.0 & 30.3 & 22.5 & 29.6 \\
\hline $\mathrm{HF}$ & 22.2 & 25.9 & 27.5 & 33.5 & 29.8 & 32.1 & 29.7 & 27.7 & 24.1 \\
\hline CACD & 8.2 & 7.8 & 18.1 & 11.6 & 10.4 & 8.5 & 14.9 & 5.2 & 20.4 \\
\hline
\end{tabular}

*Average annual mortality rates during 2002-2004 calculated by dividing the average annual number of cardiac deaths by the mean of the 2000 and 2005 populations based on the census data.

AMI, acute myocardial infarction; HF, heart failure; $\mathrm{CACD}$, cardiac arrhythmia and conduction disorders. 